\title{
Characterisation of vaccine breakthrough infections of SARS-CoV-2 Delta and Alpha variants and within-host viral load dynamics in the community, France, June to July 2021
}

\author{
François Blanquart ${ }^{1}$, Clémence Abad ${ }^{2}$, Joëvin Ambroise ${ }^{3}$, Mathieu Bernard ${ }^{4}$, Gina Cosentino ${ }^{3.5}$, Jean-Marc Giannoli ${ }^{6}$, Florence \\ Débarre $^{7}$ \\ 1. Centre for Interdisciplinary Research in Biology (CIRB), Collège de France, CNRS, INSERM, PSL Research University, Paris, \\ France* $^{*}$ \\ 2. LBM BIOESTEREL-Biogroup-Plateau technique de Mouans-Sartoux, Mouans-Sartoux, France \\ 3. BPO-BIOEPINE-Biogroup-Plateau technique Chocolaterie, Levallois-Perret, France \\ 4. BIOLITTORAL - Biogroup - Plateau technique la Bastide, Sanary sur Mer, France \\ 5. UMR1173 INSERM, Université Paris-Saclay-UVSQ, Montigny-le-Bretonneux, France \\ 6. DYOMEDEA-NEOLAB-Biogroup - Plateau technique de la Sauvegarde, Lyon, France \\ 7. Institute of Ecology and Environmental Sciences of Paris (iEES-Paris, UMR 7618), CNRS, Sorbonne Université, UPEC, IRD, \\ INRAE, 75252 Paris, France
}

Correspondence: François Blanquart (francois.blanquart@college-de-france.fr)

We compared PCR results from SARS-CoV-2-positive patients tested in the community in France from 14 June to 30 July 2021. In asymptomatic individuals, Cq values were significantly higher in fully vaccinated than non-fully vaccinated individuals (effect size: 1.7; 95\% Cl: 1-2.3; $\left.\mathrm{p}<10^{-6}\right)$. In symptomatic individuals and controlling for time since symptoms, the difference vanished $(p=0.26)$. Infections with the Delta variant had lower $\mathrm{Cq}$ values at symptom onset than with Alpha (effect size: $-3.32 ; 95 \% \mathrm{Cl}:-4.38$ to $-2.25 ; \mathrm{p}<10^{-6}$ ).

The severe acute respiratory syndrome coronavirus 2 (SARS-CoV-2) variant of concern Delta (Phylogenetic Assignment of Named Global Outbreak (Pango) lineage designation B.1.617.2 and AY.* sublineages), first detected in India, spread across the world in 2021, and in particular in Europe in late spring to early summer 2021, where it displaced the previously dominant Alpha (B.1.1.7 and Q.* sublineages) variant. Delta was shown to spread faster than Alpha [1-3] and may be associated with higher virulence [4-6] and lower vaccine effectiveness $[6,7]$ against symptomatic disease, especially with incomplete vaccination.

The Delta variant has spread in countries with high vaccination levels, and breakthrough infections have been reported, with quantification cycle $(\mathrm{Cq})$ values (also called cycle threshold (Ct)) suggesting similar or lower viral loads in vaccinated compared to unvaccinated individuals, depending on the study [8-10]. A longitudinal study has confirmed similar $\mathrm{Cq}$ values in Deltainfected vaccinated and unvaccinated individuals in the first week after diagnosis or symptom onset, with later faster decline in vaccinated individuals [11]. Comparing $\mathrm{Cq}$ values in infections with the Delta variant and infections with previous variants requires controlling for time since infection when variants have different epidemiological dynamics. This is because viral load depends on time since infection, and the distribution of time since infection across individuals depends on whether the number of cases is growing or shrinking [12-16].

Here we use a large number of PCR tests done in the community in France at a time when the Delta variant was replacing other SARS-CoV-2 strains (mostly Alpha variant) to elucidate how vaccination status, infecting variant and the presence of symptoms impact viral loads.

\section{Description of the dataset}

We studied the determinants of Cq values at the time of test and, for symptomatic individuals, as a function of the time since symptom onset, in 292,284 individuals tested from 14 June 2021 to 30 July 2021 by a large private laboratory group in the community in three regions of France (Bretagne, île-de-France, ProvenceAlpes-Côte d'Azur). These data included the result of 


\section{FIGURE 1}

Distribution of Cq values, according to vaccine status, infecting variant and symptomatic status, France, 14 June-30 July $2021(\mathrm{n}=8,437)$

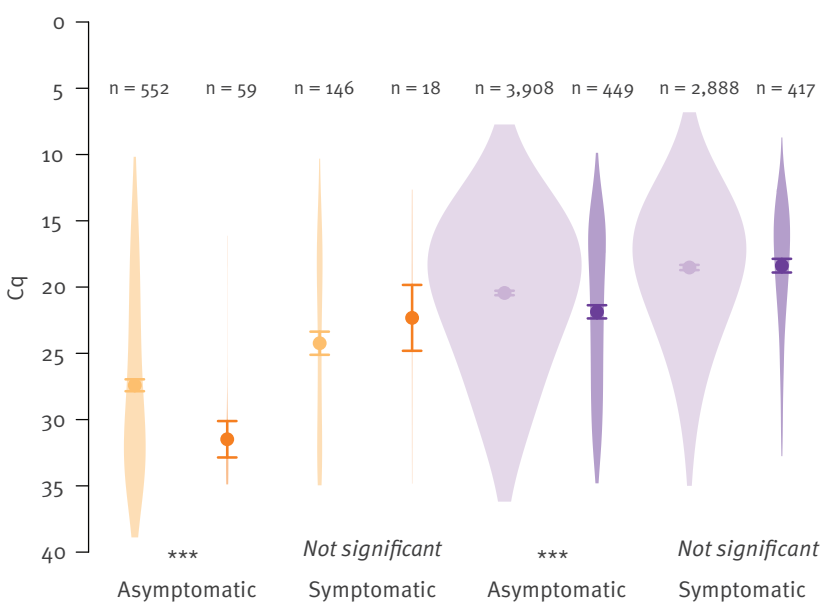

$$
\begin{array}{ll}
\text { - No vaccine, no Delta } & \text { - No vaccine, Delta } \\
\text { - Vaccine, no Delta } & \text { - Vaccine, Delta }
\end{array}
$$

Cq: quantification cycle.

\footnotetext{
$\star \star \star$ The comparison of unvaccinated and vaccinated individuals is statistically significant with $p<0.001$.
}

The widths of the violin plots reflect the number of tests; points and whiskers represent predicted values and $95 \%$ confidence intervals. Light colour: unvaccinated; dark colour: vaccinated; orange: non-Delta; purple: Delta; left side: asymptomatic at least until the test; right side: symptomatic.

the PCR test, the associated Cq value, the individual's self-reported vaccine status (fully vaccinated for at least 2 weeks or not), whether the individual has been symptomatic and the time since onset of symptoms. Positive tests were screened for the L $452 \mathrm{R}$ mutation, which characterises the Delta variant $(9,343$ positive tests had mutation information at this locus). If more than one test result was available for the same individual, we kept the last negative test if there were no positive tests, and the first positive test otherwise.

Consistent with the French vaccination campaign, vaccinated individuals in our dataset were on average (12 years older) older than non-vaccinated individuals (Supplement). The proportion of vaccinated individuals in the dataset $(24 \%)$ was smaller than in the community $(47.5 \%$ by 10 July 2021 [17]), reflecting the fact that the data are not surveillance-based.

\section{Analysis of Cq values a function of vaccination status, variant and symptoms}

We first compared the Cq values (targeted at genes RdRp and $\mathrm{N}$ ) of positive PCR tests, by vaccine status, presence of symptoms and infecting variant (Delta: presence of the $L 452 R$ mutation), for the 8,437 individuals
(3,174 female, 3,009 male, 2,254 unknown; 943 vaccinated, 7,494 unvaccinated; 6,284< 40-years-old, 2,151 $\geq 40$-years-old, two of unknown age) for whom all variables are available (Figure 1, Supplement). The $\mathrm{Cq}$ is the number of PCR cycles needed to detect a target; it is negatively correlated with viral load.

The presence of symptoms was associated with significantly lower Cq values (linear model, effect size: $\mathbf{- 2 . 7}$; 95\% confidence interval ( $\mathrm{Cl}):-3$ to -2.5 ; adjusted $\mathrm{p}<10^{-6}$, Tukey's honest significance test). Infection with the Delta variant was also associated with lower Cq values compared with non-Delta (mostly Alpha) variants (effect size: $-6.7 ; 95 \% \mathrm{Cl}:-7.1$ to -6.3 ; adjusted $\mathrm{p}\left(10^{-6}\right)$. We note that time since infection is not controlled for here, but will in the analyses further down. Vaccinated individuals had significantly higher Cq values for both Delta and non-Delta asymptomatic infections than non-vaccinated individuals (effect size: 1.7; 95\% Cl: $1-2.3$; adjusted $\mathrm{p}<10^{-6}$ ), but this difference was not significant for symptomatic infections $(p=0.8)$. For non-Delta variants, this result may be a consequence of the sample size being too small (only $n=18$ vaccinated symptomatic individuals infected with non-Delta variants).

\section{Analysis of $\mathrm{Cq}$ values controlling for the time since symptom onset}

The $\mathrm{Cq}$ values also depend on the time since infection of tested individuals. Controlling for time since infection avoids confounding factors that can arise when comparing $\mathrm{Cq}$ values of variants with different epidemiological dynamics $[13,16]$, such as Delta (increasing numbers of infections) and Alpha (decreasing numbers of infections) in the early summer of 2021 in France. As noted above, vaccinated and unvaccinated individuals may also have different test-seeking behaviour, leading to different time since infection on the day of the test. We therefore added time since symptom onset as a continuous variable in the linear model for the subset of 3,439 symptomatic infections (Supplement). We first fitted a linear model predicting $\mathrm{Cq}$ value as a function of time since symptoms, vaccination status, variant (Delta or non-Delta) and all pairwise interactions $(n=3,439)$ (Figure 2$)$. The Delta variant had a Cq difference of -3.32 on the day of symptom onset $(95 \%$ $\mathrm{Cl}:-4.38$ to -2.25 ), compared to non-Delta (Alpha) $\left(p<10^{-6}\right)$. The slope of $\mathrm{Cq}$ as a function of time was 0.6 (95\% Cl: $0.54-0.66)$ per day for Delta and 0.92 (95\% $\mathrm{Cl}: 0.73-1.1)$ for non-Delta variants $\left(p<10^{-6}\right)$. Vaccine status did not significantly alter either the Cq at symptom onset $(p=0.256)$ or the slope of $C q$ as a function of time since symptom onset $(p=0.947)$.

\section{Ethical statement}

Under French law, the use of research data that do not compromise patient privacy, as is the case with our dataset, is allowed by the French Commission Nationale Informatique et Libertés, and therefore our study did not require approval by an institutional review board. 


\section{FIGURE 2}

Regression of $\mathrm{Cq}$ values against time since symptom onset, for symptomatic individuals, by vaccine status and infecting variant, France, 14 June-30 July 2021 (n = 3,439)

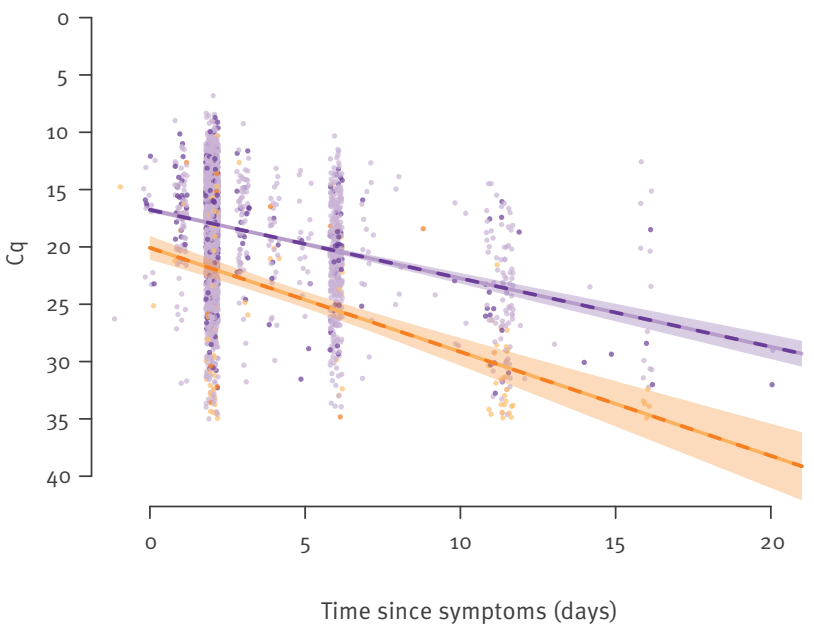

$$
\begin{aligned}
& \text { No vaccine, no Delta, . Vaccine, no Delta, } \\
& \text { No vaccine, Delta } \\
& \mathrm{n}=2,866
\end{aligned}
$$

Cq: quantification cycle.

Lines: predicted values; shaded areas: $95 \%$ confidence intervals. The vaccination effect was not significant and therefore removed from the linear model used in this figure.

\section{Discussion}

Our dataset is unique for France because variant information and vaccine status data have not been linked yet in public datasets, and quantification values are not reported at the national level (only positive/negative test results are). Our results are in line with a retrospective cohort study which found lower Cq values with Delta and longer duration of infection with low Cq [5]. Regarding vaccine breakthroughs, our results confirm studies that found similar Cq values among fully vaccinated individuals and those who were not, with the majority of infections caused by the Delta variant $[8,9]$. Two recent studies examined the viral load in vaccinated compared with unvaccinated individuals $[11,18]$. Both found that viral loads declined faster in vaccinated individuals. One study found similar viral loads in vaccinated and unvaccinated individuals until 6 days post symptom onset (or diagnosis), then faster decline in vaccinated individuals [11]. The other, a systematic longitudinal study with small sample size, found that vaccinated individuals had a faster decline in viral load already from the day of the peak [18]. The different statistical models used in the two studies may explain the difference. We did not find in our data any difference in the Cq dynamics of symptomatic vaccinated and unvaccinated individuals, but we had little statistical power to detect differences in slope late in infection as in [11]

because of a limited number of individuals presenting more than 6 days after symptom onset.

Limitations of our study stem from the way the data were collected. Reasons for seeking a SARS-CoV-2 PCR detection test are unknown, may vary among individuals and across time, and cannot be controlled for. Reasons for testing may vary between vaccinated and non-vaccinated individuals. This may especially be the case since France introduced a 'sanitary passport', requiring a proof of either full vaccination or a negative test for specific events, which may artificially inflate the proportion of negative tests among non-vaccinated individuals. Conversely, vaccinated individuals may get tested only if they have good reasons to suspect an infection. Reasons for seeking a test may also vary if symptoms differ depending on the infecting variant. Symptom and vaccine information are self-reported. Another limitation of our dataset is the lack of longitudinal data (each individual has only one Cq measure). Although our analysis takes advantage of a large sample size, additional data on randomly sampled individuals or systematic longitudinal surveys would complement our findings and shed more light on the impact of vaccination and variants on viral loads.

Finally, $\mathrm{Cq}$ values are a proxy: they are linked to viral load, and there is some evidence that viral load is associated with probability of transmission, although with considerable variation between individuals [19-21].

\section{Conclusion}

Epidemic control may require similar measures for symptomatic PCR-positive vaccinated individuals as for non-vaccinated infected individuals. This remains true even if there is indeed a late faster viral load decline in vaccinated individuals, as most transmission would already have taken place.

\section{*Authors' correction}

The affiliation 'Infection Antimicrobials Modelling Evolution, UMR1137, INSERM, Université de Paris, Paris, France' was removed for François Blanquart and the remaining affiliations were renumbered. These corrections were made on 27 October 2021 upon request from the authors.

\section{Acknowledgements}

We thank Mircea Sofonea for critical comments on a previous version of the manuscript, and Barny Young for clarifications on the Chia et al. study. FD thanks V. Glad for help keeping up with the literature.

Funding: FB was funded by a Momentum grant from the Centre National de la Recherche Scientifique. FD was funded by grant ANR-19-CE45-0009-01 from Agence Nationale de la Recherche. The funders had no role in study design, data collection and analysis, decision to publish, or preparation of the manuscript. 


\section{Conflict of interest}

None declared.

\section{Authors' contributions}

FD and FB conceived the study, analysed the data, wrote the paper, and should be considered as co-corresponding authors. CA, JA, MB, GC, JMG collected the data and commented on the paper.

\section{References}

1. Campbell F, Archer B, Laurenson-Schafer H, Jinnai Y, Konings F, Batra N, et al. Increased transmissibility and global spread of SARS-CoV-2 variants of concern as at June 2021. Euro Surveill. 2021;26(24):2100509. https://doi.org/10.2807/1560-7917. ES.2021.26.24.2100509 PMID: 34142653

2. Mlcochova P, Kemp S, Dhar MS, Papa G, Meng B, Ferreira IATM, et al. SARS-CoV-2 B.1.617.2 Delta variant replication and immune evasion. Nature. 2021. https://doi.org/10.1038/ S41586-021-03944-y PMID: 34488225

3. Alizon S, Haim-Boukobza S, Foulongne V, Verdurme L, Trombert-Paolantoni S, Lecorche E, et al. Rapid spread of the SARS-CoV-2 Delta variant in some French regions, June 2021. Euro Surveill. 2021;26(28):2100573. https:// doi.org/10.2807/1560-7917.ES.2021.26.28.2100573 PMID: 34269174

4. Fisman DN, Tuite AR. Progressive increase in virulence of novel SARS-CoV-2 variants in Ontario, Canada. medRxiv 2021.07.05.21260050. Preprint. http://dx.doi.org/ https://doi. org/10.1101/2021.07.05.21260050

5. Ong SWX, Chiew CJ, Ang LW, Mak T-M, Cui L, Toh MPHS, et al. Clinical and virological features of SARS-CoV-2 variants of concern: a retrospective cohort study comparing B.1.1.7 (Alpha), B.1.315 (Beta), and B.1.617.2 (Delta). Clin Infect Dis. 2021;ciab721. PMID: 34423834

6. Sheikh A, McMenamin J, Taylor B, Robertson C, Public Health Scotland and the EAVE II Collaborators. SARS-CoV-2 Delta VOC in Scotland: demographics, risk of hospital admission, and vaccine effectiveness. Lancet. 2021:397(10293):2461. 2. https://doi.org/10.1016/S0140-6736(21)01358-1 PMID: 34139198

7. Lopez Bernal J, Andrews N, Gower C, Gallagher E, Simmons R, Thelwall S, et al. Effectiveness of Covid-19 Vaccines against the B.1.617.2 (Delta) Variant. N Engl J Med. 2021;385(7):585-94. https://doi.org/10.1056/NEJMoa2108891 PMID: 34289274

8. Brown CM, Vostok J, Johnson H, Burns M, Gharpure R, Sami S, et al. Outbreak of SARS-CoV-2 infections, including COVID-19 vaccine breakthrough infections, associated with large public gatherings - Barnstable County, Massachusetts, July 2021. MMWR Morb Mortal Wkly Rep. 2021;70(31):1059-62. https:// doi.org/10.15585/mmwr.mm7031e2 PMID: 34351882

9. Public Health England (PHE). SARS-CoV-2 variants of concern and variants under investigation in England. Technical briefing 20. London: PHE; 2021. Available from: https:// assets.publishing.service.gov.uk/government/uploads system/uploads/attachment_data/file/1009243/Technical Briefing_20.pdf

10. Elliott P, Haw D, Wang H, Eales O, Walters CE, Ainslie KEC, et al. REACT-1 round 13 final report: Exponential growth, high prevalence of SARS-CoV-2 and vaccine effectiveness associated with Delta variant in England during May to July 2021. London: Imperial College; 2021. Available from: https:// spiral.imperial.ac.uk/bitstream/10044/1/90800/2/react1_r13_ final_preprint_final.pdf

11. Chia PY, Ong S, Chiew CJ, Ang LW, Chavatte JG, Mak TM, et al. Virological and serological kinetics of SARS-CoV-2 Delta variant vaccine-breakthrough infections: A multi-center cohort study. medRxiv. 2021.07.28.21261295. Preprint. http://dx.doi. org/https://doi.org/10.1101/2021.07.28.21261295

12. Alizon S, Selinger C, Sofonea MT, Haim-Boukobza S, Giannoli $J-M$, Ninove L, et al. Epidemiological and clinical insights from SARS-COV-2 RT-PCR cycle amplification values. medRxiv. 2021.03.15.21253653. Preprint. http://dx.doi.org/ https://doi. org/10.1101/2021.03.15.21253653

13. Hay JA, Kennedy-Shaffer L, Kanjilal S, Lennon NJ, Gabriel SB, Lipsitch M, et al. Estimating epidemiologic dynamics from cross-sectional viral load distributions. Science. 2021;373(6552):eabho635. https://doi.org/10.1126/science. abho635 PMID: 34083451
14. Cosentino G, Bernard M, Ambroise J, Giannoli J-M, Guedj J, Débarre F, et al. SARS-CoV-2 viral dynamics in infections with Alpha and Beta variants of concern in the French community. Infect. 2021;S0163-44532100374-1. PMID: 34329672

15. Althaus CL, Baggio S, Reichmuth ML, Hodcroft EB, Riou J, Neher RA, et al. A tale of two variants: Spread of SARS-CoV-2 variants Alpha in Geneva, Switzerland, and Beta in South Africa. medRxiv. 2021.06.10.21258468. Preprint. http://dx.doi. org/ https://doi.org/10.1101/2021.06.10.21258468

16. Hay JA, Kennedy-Shaffer L, Mina MJ. Viral loads observed under competing strain dynamics. medRxiv. 2021.07.27.21261224. Preprint. http://dx.doi.org/ https://doi. org/10.1101/2021.07.27.21261224

17. Santé Publique France. Données relatives aux personnes vaccinées contre la Covid-19. [Data relating to people vaccinated against Covid-19]. Paris: French Government. [Accessed: 2021-08-09]. French. Available from: https://www. data.gouv.fr/en/datasets/donnees-relatives-aux-personnesvaccinees-contre-la-covid-19-1/

18. Kissler SM, Fauver JR, Mack C, Tai CG, Breban MI, Watkins AE, et al. Viral dynamics of SARS-CoV-2 variants in vaccinated and unvaccinated individuals. medRxiv. 2021.02.16.21251535 Preprint. http://dx.doi.org/ https://doi.org/10.1101/2021.02.1 6.21251535

19. Lee LYW, Rozmanowski S, Pang M, Charlett A, Anderson C, Hughes GJ, et al. SARS-CoV-2 infectivity by viral load, S gene variants and demographic factors and the utility of lateral flow devices to prevent transmission. Clin Infect Dis. 2021; ciab421. PMID: 33972994

20. Marc A, Kerioui M, Blanquart F, Bertrand J, Mitjà O, Corbacho-Monné $M$, et al. Quantifying the relationship between SARS-CoV-2 viral load and infectiousness. medRxiv. 2021.05.07.21256341. Preprint. http://dx.doi.org/ https://doi. org/10.1101/2021.05.07.21256341

21. Lyngse FP, Mølbak K, Træholt Franck K, Nielsen C, Skov RL, Voldstedlund M, et al. Association between SARS-CoV-2 transmissibility, viral load, and age in households. medRxiv. 2021.02.28.21252608. Preprint. http://dx.doi.org/ https://doi. org/10.1101/2021.02.28.21252608

\section{License, supplementary material and copyright}

This is an open-access article distributed under the terms of the Creative Commons Attribution (CC BY 4.0) Licence. You may share and adapt the material, but must give appropriate credit to the source, provide a link to the licence and indicate if changes were made.

Any supplementary material referenced in the article can be found in the online version.

This article is copyright of the authors or their affiliated institutions, 2021. 